\title{
Arzthelferin schwanger - was ist zu tun?
}

Sobald eine Arbeitnehmerin ihren Arbeitgeber darüber informiert, dass sie schwanger ist, treten eine Reihe gesetzlicher Bestimmungen in Kraft, die das Wohl der werdenden Mutter gewährleisten und Gefährdungen für das ungeborene Kind verhindern sollen. Ein Urteil des Bundesverwaltungsgerichts aus den 1990er-Jahren hat dabei besondere Maßstäbe für den Bereich des Gesundheitswesens angesetzt. Demnach dürfen schwangere Frauen in Arztpraxen von Anfang an nicht mit infektiösen Materialien hantieren (Az.: 5 C 42/89).

Konkret bedeutet das für den täglichen Praxisbetrieb, dass werdende Mütter weder mit einer Lanzette den Blutzucker messen noch Injektionen verabreichen dürfen. Diese Einschränkungen, die sich auch auf zahlreiche weitere Tätigkeiten erstrecken, gelten selbst dann, wenn die Helferin mit Handschuhen ar-

beitet. Streng genommen bedeutet das, dass die Mitarbeiterin gar nicht mehr am Patienten arbeiten darf, sondern z.B. nur noch an der Anmeldung.

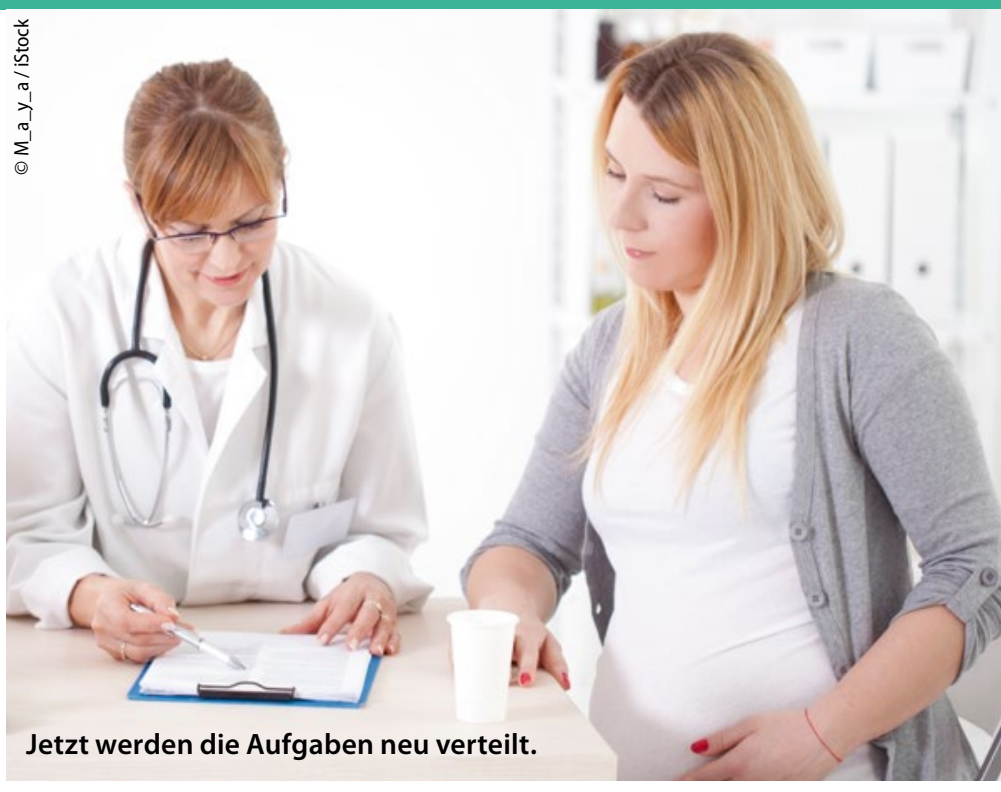

\section{MMW-KOMMENTAR}

Mehrarbeit (Überstunden) oder Arbeiten zwischen 20 und 6 Uhr sowie an Sonn- und Feiertagen sind bei Schwangerschaft von Anfang an verboten. Schwangere dürfen maximal 8,5 Stunden pro Tag oder 90 Stunden pro Doppelwoche arbeiten, bei Minderjährigen sind es 8 bzw. 80 Stunden. Fahrzeiten zur Arbeit und Pausen werden nicht berücksichtigt. Die durch ein Beschäftigungsverbot entstehenden Lohnausfallskosten werden von der Krankenkasse erstattet. Lediglich wenn die schwangere Mitarbeiterin erkrankt, gilt die sechswöchige Lohnfortzahlungspflicht. Grundsätzlich gilt das Beschäftigungsverbot spätestens ab der 34. Schwangerschaftswoche. Es endet acht Wochen nach Geburt, bei Früh-oder Mehrlingsgeburten sind es zwölf Wochen. Schließt sich die Elternzeit nahtlos an den Mutterschutz an, trägt die Krankenkasse auch hier die Lohnausfallkosten. Die Stelle kann in dieser Zeit neu besetzt werden, muss aber der Arzthelferin am Ende der Elternzeit wieder zur Verfügung stehen.

\section{Auch bei Hilfsmitteln gibt es Regressgefahr}

Die gesetzlichen Krankenkassen legen in einem Verzeichnis fest, welche verordneten Hilfsmitteln sie bezahlen. Die Liste enthält alle Produkte, deren Kosten erstattet werden - aktuell etwa 20.000 Artikel. Sie wird fortlaufend aktualisiert und hat einen Empfehlungscharakter. Im juristischen Sinne ist sie deshalb nicht bindend, es dürfen also auch Produkte verordnet werden, die nicht auf der Liste stehen. Hilfsmittel werden auf dem Muster 16 verordnet, das auch für die Verordnung von Arzneimitteln Verwendung findet. Für Hörund Sehhilfen sowie vergrößernde Sehhilfen gibt es dagegen eigene Muster.
Das Hilfsmittel muss so eindeutig wie möglich bezeichnet werden. Das Rezept muss alle für die individuelle Versorgung oder Therapie notwendigen Angaben enthalten. Dazu gehören die Diagnose, das Verordnungsdatum und die Bezeichnung des Hilfsmittels laut Verzeichnis, falls es dort gelistet ist.

\section{MMW-KOMMENTAR}

Die Hilfsmittelverordnung unterliegt nicht der Wirtschaftlichkeitsprüfung nach \$106 SGB V. Das schützt aber nicht vor Regressen im Gegenteil: Streng genommen ist die Regressgefahr hier sogar größer als bei der Verordnung von Arznei- und Heilmitteln. Ein
Regress ist dann möglich, wenn ein Hilfsmittel verordnet wurde, das nicht auf der Hilfsmittelliste steht, oder wenn nicht der kostengünstigste Weg der Verordnung gewählt wurde.

Auf einem Hilfsmittelrezept sollte deshalb grundsätzlich „Nur nach Genehmigung durch die Krankenkasse" vermerkt werden. Der Patient sollte sich damit zunächst an seine Kasse wenden, die ihm einen Hilfsmittelanbieter nennt, mit dem sie einen Versorgungsvertrag hat. Wurde ein Hilfsmittel individuell angefertigt, so ist nach der Anpassung zu prüfen, ob das Hilfsmittel der Verordnung entspricht, korrekt angefertigt ist und seinen Zweck erfüllt. 